\title{
Stephan Leibfried Die Institutionalisierung der Arbeitslosenversicherung in Deutschland
}

In dieser Untersuchung sollen einige gesellschaftliche Rahmenbedingungen für die Institutionalisierung einer Arbeitslosenversicherung in Deutschland herausgearbeitet werden. Nach einer Skizze der einzelnen historischen Entwicklungsschritte soll das spezifisch riskante dieser Versicherungsstruktur im Bereich der sozialen Sicherung vorgestellt und versucht werden, die historische Verspätung in der Errichtung gerade dieses Versicherungszweiges zu erklären.

\section{Historische Entwicklungsschritte ${ }^{1}$}

In Deutschland entwickelt sich in drei großen Etappen eine spezifische positive Form der öffentlichen Absorbtion des Risikos der "kapitalistischen « Arbeitslosigkeit (Arbeitslosigkeit als ein Gegenstand unter anderen der allgemeinen Armenpflege; Arbeitslosigkeit als Gegenstand eines besonderen, privilegierten Fürsorgezweiges; Arbeitslosigkeit als Gegenstand eines gesonderten, versicherungsförmigen $\mathrm{Be}$ rechtigungssystems). Dieses Risiko wird erst im 20. Jahrhundert isoliert und eigenständigen, relativ ausdifferenzierten Bearbeitungsformen unterworfen. So wird zunächst schrittweise eine eigene Erwerbslosenfürsorge aufgebaut und diese 1927 in eine Arbeitslosenversicherung überführt.

Die Grundstufen dieser Entwicklung seien kurz skizziert:

I8. I2. I914: Der Bundesrat erläßt Bestimmungen zur Durchführung der Kriegswohlfahrtspflege (Bekanntmachung in RZBl 1914, 619 f.). Hiernach subventioniert das Reich die Erwerbslosenausgaben der Gemeinden. Im übrigen werden für spezielle Industriezweige Sonderfürsorgen geschaffen (z. B. Textil). (Etabilierung einer herausgebobenen Fürsorge für Erwerbslose).

I3. I I. I9 18: Nach der Verordnung über die Erwerbslosenhilfe (RGB1. I305) sind die Gemeinden nunmehr alle zur Einrichtung einer Erwerbslosenfürsorge verpflichtet. Die Gemeinden erhalten Kostenersatz vom Reich in Höhe von $\%$ und von dem jeweiligen Land in Höhe von $\%$ der Kosten. Arbeitnehmerorganisationen werden in die Ausführung der Fürsorge integriert (Etablierung der Gruppenmitwirkung bei der Vergabe materieller Unterstützung).

5. 5. 1920: Einrichtung eines Reichsamtes für Arbeitsvermittlung zur Beobachtung des Arbeitsmarkts und zur Gewährleistung einer einheitlichen

\footnotetext{
I Zur geschichtlichen Entwicklung der übrigen Träger der Sozialversicherung vgl. Tennstedt 76,385 ff. Zu einem, auch historisch angelegten, Ubberblick zur Sozialhilfe vgl. Leibfried 77, $9 \mathrm{ff}$.
} 
Regelung der Arbeitsvermittlung (Etablierung eines organisatorischen Nukleus für eine Arbeitslosenversicherung im Zentralstaat).

22. 6. 1922: Durch das Arbeitsnachweisgesetz (RGBI. I, 657) werden die Gemeinden verpflichtet, Arbeitsnachweise zu errichten. Den Arbeitsnachweisen obliegt die Mitwirkung bei der Durchführung von gesetzlichen Unterstützungsmaßnahmen (Etablierung einer an das Unterstützungswesen rückgekoppelten Arbeitsvermittlung).

15. 10. 1923: Die Verordnung über die Aufbringung der Mittel für die Erwerbslosenfürsorge (RGBI. I, 984) aufgrund des Ermächtigungsgesetzes vom 13. 10. 1923 (BGBl. I, 943) schreibt eine Beitragspflicht von Arbeitgebern und Arbeitnehmern in gleicher Höhe zu den Kosten der Erwerbslosenfürsorge vor. Die Höhe wird jeweils für den Bezirk von dem Verwaltungsausschuß des öffentlichen Arbeitsnachweises festgelegt. Die Festlegung erfolgt in Bruchteilen des Beitrages zur Krankenversicherung (mit einem Maximum von $20 \%$ ) und der Einzug erfolgt durch die Krankenkasse. Der Ertrag soll $/ /$ der Aufwendungen der Gemeinde für die Erwerbslosenfürsorge decken. Die Gemeinde trägt 1/s selbst. (Parafiskalisierung der Arbeitslosenunterstïtzung auf der Grundlage paritätischer Beiträge).

16. 7. 1927: Der Reichstag beschließt das Gesetz über Arbeitsvermittlung und Arbeitslosenversicherung (AVAVG) nach mehriähriger Beratung über verschiedene Entwürfe (RGBl. I, 187). Das Gesetz sieht eine "Reichsanstalt für Arbeitsvermittlung « vor, deren Aufgaben Arbeitsvermittlung, Arbeitslosenversicherung, öffentliche Berufsberatung und Lehrstellenvermittlung ( $\$$ I I, II I) sind. "Weitere Aufgaben zur Regelung des Arbeitsmarkts « kann sie mit Zustimmung des Reichsarbeitsministers übernehmen. Das Gesetz sieht eine Gruppenmitwirkung $(\mathbb{S} S \mathrm{ff}$.) und eine parafiskalische Finanzierung $(\mathbb{S} 142 \mathrm{ff}$.) vor. (Vgl. Weller 69, 34 ff.; Wermel/Urban 49 II; Preller 49; vgl. allgemein auch Stolleis 76).

Angesichts eines bei Beschluß des AVAVG dominierenden Uberangebots an Arbeitskräften war das Handlungsinstrumentarium der Reichsanstalt zunächst durch das Vorherrschen eines Kernbestands an Möglichkeiten für eine passive Arbeitsmarktsanierung gekennzeichnet, ein Bestand der allerdings zudem auf konjunkturelle - und nicht auch auf strukturelle - Krisen zugeschnitten war. Den Arbeitsmarkt passiv zu sanieren heißt, sich allein auf die informatorische Verflüssigung der "Zufuhr" und der Milderung von Friktionen im Markt bzw. bei der »Abfuhr « zu konzentrieren, also die Bewegungen des Arbeitsmarktes grundsätzlich noch als naturwüchsige hinzunehmen und bei dem Ausgleich der Folgen dieser Bewegungen anzusetzen. So organisierte die Anstalt Arbeitsvermittlung, Berufsberatung und Lehrstellenvermittlung; sie leistete Arbeitslosengeld, Arbeitslosenhilfe und Kurzarbeitergeld und förderte finanziell die Arbeitsaufnahme; zudem konnte die Anstalt Notstandsarbeiten unterstützen (vgl. Weller 69, 49 ff.). In einem weiteren Ausdifferenzierungs- und Spezifizierungsschritt wird das Handlungsinstrumentarium der zuständigen Körperschaft

- auch auf saisonale Arbeitslosigkeit und zwar durch ergänzende materielle Förderung zugeschnitten: Förderung der ganzjährigen Beschäftigung in der Bauwirtschaft seit 1959, jetzt $\$ \$ 74-89$ AFG (vgl. Weller 69, 20r ff.);

- für besonders schwer zu vermittelnde Problemgruppen durch Ergänzung der materiellen Förderungsmöglichkeiten angereichert: Maßnahmen zur Arbeitsbeschaffung für ältere Arbeitnehmer, jetzt $\$ \$ 97-99$ AFG; 
- in einem besonderen Problembereich um ergänzende materielle Förderung wie auch um die intensive Förderung von Qualifizierung vervollständigt: Rehabilitation, jetzt $\$ \$ 56-62$ AFG und ergänzendes Recht.

Schließlich wird in einem Zeitraum, der deutlich durch einen Wechsel zwischen Uberfluß und Knappheit an Arbeitskräften bestimmt ist - ein Wechsel der wiederum durch eine u. a. qualifikationsbestimmte Aufsplitterung des Arbeitsmarktes in Untergruppen kompliziert wird -, ein umfassender Ausbau der Förderung von Maßnahmen der beruflichen Bildung zu einem gesetzlichen Bestandteil der Arbeitslosenversicherung gemacht (1969). Aus diesem Anlaß wird das AVAVG in das Arbeitsförderungsgesetz (AFG) umbenannt. Damit wird Qualifikationspolitik zu einem Mittel von Prävention, Marktflexibilität und "Aufbewahrung" auch für die schon aktive Arbeitsbevölkerung. Dieses Maßnahmeinstrumentarium institutionalisiert damit den "quartären « Bildungssektor und vervollständigt die staatliche sozialisative Intervention.

II. Was ist das spezifische Risiko der Arbeitslosenversicherung im System der sozialen Sicherung?

Die Arbeitslosenversicherung wird rechtssystematisch oder sozialpolitisch-typologisch oft nur als ein Element in der Vielzahl der sozialen Versicherungen beschrieben und begriffen, so z. B. in der üblichen Trias Versicherung-Versorgung-Fürsorge (anders die $\mathbb{S}, 4$ SGB-AT).

Diese Zuordnung ist nicht nur deshalb zweifelhaft geworden, weil im Laufe der Entwicklung der Bundesrepublik die Aufgaben zunehmen relativiert worden sind, die als eigentümliche Versicherungsaufgaben der Bundesanstalt angesehen wurden. Eine solche Relativierung war vor allem zeitweise zugunsten der beruflichen Bildung festzustellen. Eine solche Zuordnung verbirgt auch, daß der soziale Ort dieser Institution in der gesellschaftlichen Reproduktion ein durchaus eigenartiger und hervorstechender ist, gerade wenn man auf einen Vergleich mit den anderen Versicherungen abstellt. Nur die Arbeitslosenversicherung betrifft ausschließlich "den ökonomischen Ort, an welchem der Arbeiter mit den übrigen Klassen der Gesellschaft zusammenhängt«, den Arbeitmarkt (vgl. Lederer/Marschak 27, II2). Deshalb umfaßt die Arbeitslosenversicherung auch nicht die speziellen gebrauchswertorientierten Folgerisiken, die sich auch dem vermögenslosen und einkommensabhängigen Status eines Arbeitnehmers ergeben (Krankheit, Alter, Unfall). Sie thematisiert vielmehr diesen riskanten Status selbst. Deshalb ist auch im Fall der Arbeitslosenversicherung die konkrete Bestimmung des Versicherungsfalls schwieriger und brisanter als in den anderen Versicherungszweigen. Geht es in den anderen Versicherungszweigen in der Regel nur um relativ einfache, objektivierbare, reale Umstände (wie Krankheit, Alter, Erwerbsunfähigkeit), die oft aufgrund ihrer vergleichsweise geringeren Brisanz in vorbürokratischer Weise privat festgestellt werden (Urteil des behandelnden Arztes), so geht es in jenem Fall der Arbeitslosenversicherung um subjektive Lagen einerseits ${ }^{2}$ und um komplexere soziale Objektivierungen andererseits ${ }^{3}$. 4, die wegen ihrer brisanten Nähe zum Arbeitsmarkt

2 Arbeitslos ist u. a. nur, wer bereit ist, jede zumutbare Beschäftigung anzunehmen (vgl. $\ 103$ Abs. 1 AFG); vgl. auch die $\$ \$ 19$ AFG, 66 AT-SGB.

3 Arbeitslos ist u. a. nur, wer eine zumutbare Beschäftigung unter den Bedingungen des allgemeinen Arbeitsmarktes ausüben kann, $\$ 103$ Abs. I AFG. Vgl. im übrigen die Zugangsbedingungen zur beruflichen Bildung, $\$ \$ 40 \mathrm{ff}$. AFG.

${ }_{4} \mathrm{Vgl}$. insoweit auch BVerfGE 18, 366, 376: "Außerdem sind die Schadensfälle der anderen (Versicherungs-St. L.) Zweige im wesentlichen durch objektive Merkmale, wie Arbeitsunfähigkeit durch Krank- 
ausschließlich Gegenstand von Bearbeitung in der Form bürokratischer Herrschaft sind.

Aufgrund dieses sozialen Orts und des anderen Zuschnitts der konkreten Bestimmungen des Versicherungsfalles haben die Verfahren unmittelbar bürokratischer Verhaltenskontrolle in diesem Versicherungszweig einen Stellenwert, der ihnen in anderen Versicherungszweigen nicht zukommt. Insoweit braucht nur auf die $\$ \mathbb{1 0 3}$, I 9 AFG sowie auf die Arbeitsvermittlung und Berufsberatung hingewiesen werden. Es bestehen hier quasi sozialarbeiterische Kontaktzwänge zwischen Bürokratie und Klientel. Es finden sich daher auch die entsprechenden Brüche zu denen der bürokratischen Organisation von Sozialarbeit: der Trennung von Innendienst (materielle Leistungen der Sozialhilfe) und Außendienst (Sozialarbeit) im Sozialamt entspricht die in den Innendienst hinein gelagerte Trennung von Leistungsabteilung und den Abteilungen Arbeitsvermittlung sowie Berufsberatung des Arbeitsamts. Alle anderen Versicherungszweige haben selbst nur ein distanziert-schriftliches, aktenmäßiges, bescheidorientiertes Verhältnis zu den Leistungsberechtigten. Etwaige Kontaktzwänge sind durch Auslagerung in den medizinisch professionalisierten Sektor aus dem bürokratischen Sektor ausgeschieden. Der diese klassischen bürokratischen Strukturen modifizierende Zuschnitt der Arbeitslosenversicherung ist nur eine Funktion des Zwangs, sich konkret auf den Arbeitsmarkt zu orientieren, ihres Zuschnitts als konkreter "Regulierer" der Flüssigkeit bzw. Fixiertheit von Arbeitskräften. Um den Sog des Kontaktzwangs auszugleichen und um den nur in nationalem Maßstab realisierbaren Regulierungsfunktionen nachzukommen hat die Arbeitslosenversicherung schließlich auch - vor allem im Vergleich zur Unfall- und Krankenversicherung - eine stark durchgebildete, standardisierte, zentralisierte und hierarchische Organisationsstruktur. Sie kommt also der klassischen Organisation einer Staatsbehörde insoweit noch am nächsten, damit sie ihre arbeitsmarktpolizeilichen Aufgaben wirksam erfüllen kann.

III. Welche strukturellen Hindernisse begründen die bistorisch späte Institutionalisierung der Arbeitslosenversicherung?

Von den anderen Institutionen der sozialen Versicherung hebt sich die Arbeitslosenversicherung auch dadurch $a b$, daß sie zu einem relativ späten Zeitpunkt entsteht. Sie wurde durch das AVAVG vom I6. Juli 1927 geschaffen. Ihr Entstehen war allerdings durch einen schrittweisen Aufbau der Erwerbslosenfürsorge vorbereitet. Ein Blick auf Schaubild I zeigt, daß der geringste zeitliche Abstand zwischen der Errichtung der Arbeitslosenversicherung und dem Aufbau der anderen Zweige sozialer Sicherung in Deutschland 38 Jahre beträgt.

Daß diese historische Verspätung nicht zufällig ist, läßt sich zunächst plausibel machen, wenn die Entstehungszeiten der vier Zweige sozialer Sicherung in zwölf kapitalistischen, westeuropäischen Gesellschaftsordnungen systematisch näher betrachtet werden. Schaubild I enthält auch das für diesen Vergleich nötige Datenmaterial: die Entstehungsdaten für zwangsförmige und freiwillige Versicherungsformen in den vier Bereichen sozialer Sicherung. Diese Daten lassen sich in folgender Weise ordnen:

- Es können Häufigkeitsverteilungen in der Reihenfolge der Entstehung der vier Zweige sozialer Sicherung ermittelt werden (vgl. Tabelle I, Reihenfolge der Einführung: Ränge);

heit oder Unfall, sowie Invalidität und Alter feststellbar; der Eintritt der Arbeitslosigkeit hängt dagegen auch weitgehend von dem subjektiven Verhalten der Arbeitgeber und Arbeitnehmer ab." 
Schaubild ${ }_{I}$

Zentrale Gesetzgebungswerke im Bereich der sozialen Sicherheit in Westeuropa

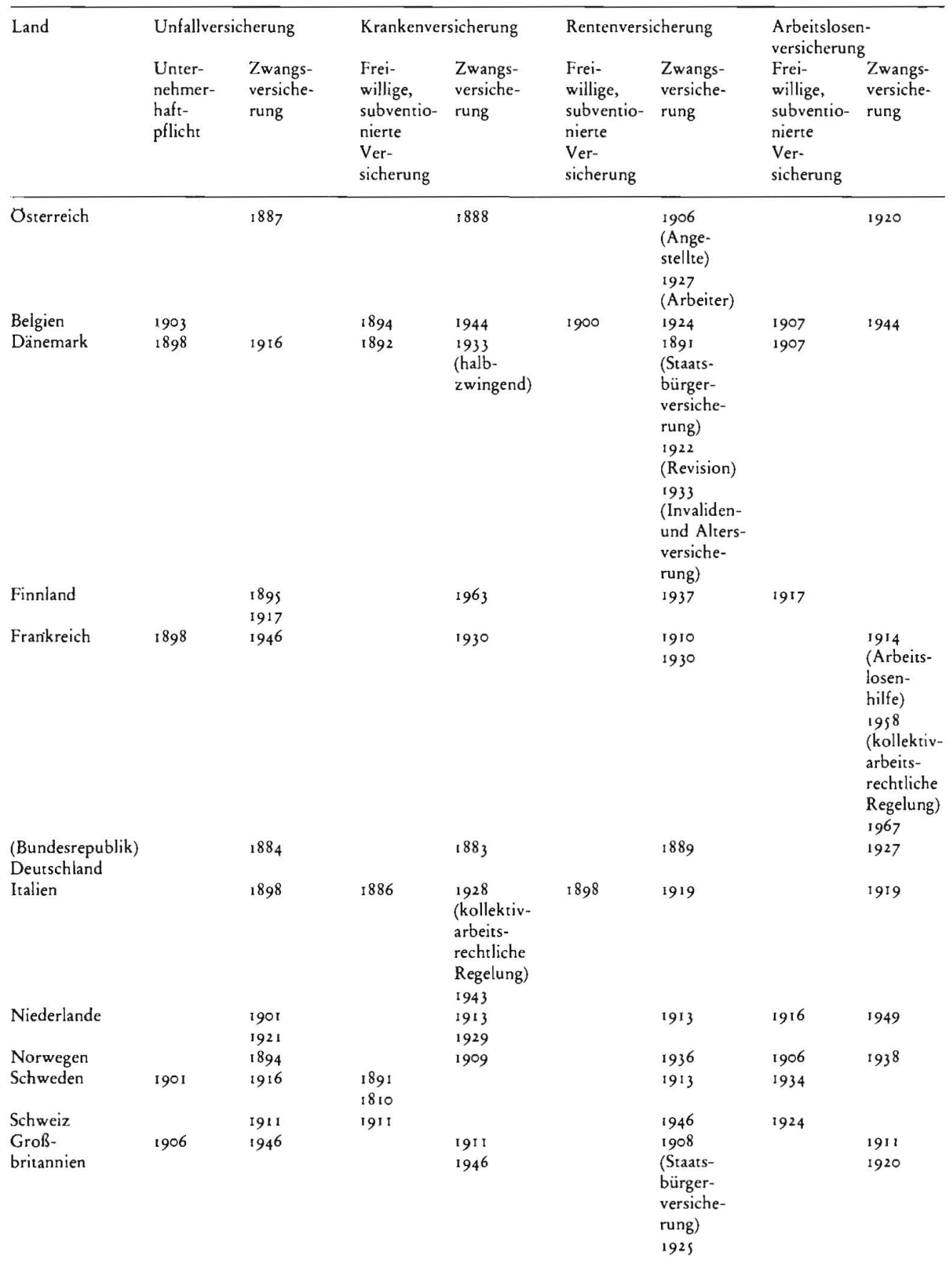

(Quelle: Flora 76, 5i) 
- Der Zeitpunkt der Institutionalisierung sozialpolitischer Reform kann bestimmt werden

- als durchschnittlicher Zeitpunkt für alle I2 Länder;

- als Markierung der Extrempunkte: früheste und späteste Einführung in der Gruppe der 12 Länder

(vgl. Tabelle I, Jahr der Einführung).

Tabelle $I$

Zur Systematik in der Reihenfolge der Entstehung von Systemen der sozialen Versicherung

\begin{tabular}{|c|c|c|c|c|c|c|c|c|}
\hline & \multicolumn{5}{|c|}{ Reihenfolge der Einführung: Ränge } & \multicolumn{3}{|c|}{ Jahr der Einführung } \\
\hline & 1. & 2. & 3 . & 4. & $\begin{array}{l}\text { Durch- } \\
\text { schnitt }\end{array}$ & frühestes & spätestes & $\begin{array}{l}\text { Durch- } \\
\text { schnitt }\end{array}$ \\
\hline Unfallversicherung & $\begin{array}{l}7 \\
(6)\end{array}$ & $\begin{array}{c}2 \\
(4)\end{array}$ & $\begin{array}{c}1 \\
(2)\end{array}$ & $\stackrel{2}{(-)}$ & $\begin{array}{c}1.8 \\
(1.7)\end{array}$ & $\begin{array}{c}1884 \\
(1884)\end{array}$ & $\begin{array}{c}1971 \\
(1911)\end{array}$ & $\begin{array}{r}1914 \\
(1898)\end{array}$ \\
\hline Krankenversicherung & $\begin{array}{l}2 \\
\text { (s) }\end{array}$ & $\begin{array}{c}4 \\
(2)\end{array}$ & (3) & $\begin{array}{l}1 \\
(2)\end{array}$ & $\begin{array}{c}2.4 \\
(2.2)\end{array}$ & $\begin{array}{c}1883 \\
(1883)\end{array}$ & $\begin{array}{c}1963 \\
(1963)\end{array}$ & $\begin{array}{r}1923 \\
(1906)\end{array}$ \\
\hline Rentenversicherung & $\begin{array}{l}3 \\
\text { (1) }\end{array}$ & (4) & $\begin{array}{l}3 \\
\text { (s) }\end{array}$ & $\begin{array}{c}1 \\
\text { (2) }\end{array}$ & $\begin{array}{c}2.2 \\
(2.7)\end{array}$ & $\begin{array}{c}1889 \\
(1889)\end{array}$ & $\begin{array}{c}1946 \\
(1946)\end{array}$ & $\begin{array}{r}1922 \\
(1912)\end{array}$ \\
\hline Arbeitslosenversicherung & $\begin{array}{l}- \\
(-)\end{array}$ & $\begin{array}{l}1 \\
\text { (2) }\end{array}$ & $\begin{array}{l}3 \\
(2)\end{array}$ & $\begin{array}{c}8 \\
(8)\end{array}$ & $\begin{array}{r}3.6 \\
(3.5)\end{array}$ & $\begin{array}{c}1911 \\
(1906)\end{array}$ & $\begin{array}{c}1967 \\
(1934)\end{array}$ & $\begin{array}{c}1930 \\
(1917)\end{array}$ \\
\hline
\end{tabular}

Diese vergleichende, systematische Betrachtungs zeigt, daß die Verspätung kein zufälliges, sondern ein strukturelles Phänomen sein dürfte:

- Im allgemeinen ist zuerst die Unfallversicherung und zuletzt die Arbeitslosenversicherung eingeführt worden. Bei den Aussagen über die Reihenfolge der Institutionalisierung hat diejenige über die Letztentstehung der Arbeitslosenversicherung sogar die höchste Wahrscheinlichkeit für sich. In acht von zwölf Fällen entstand die Arbeitslosenversicherung als letzte. Demgegenüber entstand die Unfallversicherung nur in sieben von zwölf Fällen als erste.

- Eine zwingende Arbeitslosenversicherung ist zuerst in England I91 I eingeführt worden und zuletzt 1967 in Frankreich. Der durchschnittliche Zeitpunkt der Einführung liegt um 1930. Die Einführung der Arbeitslosenversicherung in Deutschland liegt also ziemlich nahe am Durchschnittspunkt.

Wie läßt sich diese Verspätung als strukturell bedingt erklären? Bei dem Versuch, die Arbeitslosenversicherung zu institutionalisieren, mußte eine Vielzahl von Hindernissen überwunden werden. Es handelt sich um Hindernisse, welche in ihrer Gesamtheit nur beim Aufbau einer Arbeitslosenversicherung nicht aber bei der Errichtung der anderen Versicherungsarten zu überwinden waren. Zur Ưberwindung dieser Hindernisse war ein besonderer zeitlicher und sachlicher Institutionalisierungsaufwand erforderlich. Die Erklärung der zeitlichen Verzögerung aus Hindernissen kann allerdings nur die Verspätung als solche, nicht jedoch erklären, warum das AVAVG gerade 1927 beschlossen wurde. An diesem Punkt muß eine funktionale Analyse notwendig versagen und kann allein eine historisch-empirische, auf Kontingenz zielende Untersuchung weiter führen (vgl. Goldthorpe 62, 53 ff.).

\footnotetext{
5 Bei dieser Betrachtung fällt auch auf, daß nur Deutschland und Osterreich ausschließlich Versicherungsformen mit $Z$ wangsmitgliedschaft aufgebaut haben. Dieser besondere Zuschnitt der deutschen Sozialpolitik mag der speziellen Staatstradition Deutschlands (etatistische Staatsfürsorge) zu verdanken sein. Zur Behandlung der Arbeitslosen in einigen der kapitalistischen Kernländer vgl. für die USA Lubove 68, Nelson 69 und Stewart 76, für England Gilbert 66 und 70 sowie Mesher 76, für Frankreich Fournier/ Questiaux 76 und zu einem breiteren, verg)eichenden Úberblick Schmid 75 (für die Länder Frankreich, Großbritannien, Schweden, Bundesrepublik Deutschland, DDR, UdSSR).
} 
Folgende sieben Hindernisse können der Errichtung öffentlicher Systeme sozialer

Sicherung entgegenstehen:

(I) Bruch mit der sozialstrukturellen Individualisierung von an sich gesellschaftlichen Risiken

(2) mangelndes Äquivalent zu bestehenden Rechtsformen

(3) Finanzwirksamkeit der Maßnahmen

(4) Reichtum an sozialen Voraussetzungen, nämlich einerseits, einem entwickelten nationalen Arbeitsmarkt, und andererseits, einer Umdefinition der öffentlichen Hand zu einem positiven Garanten der kapitalistischen Entwicklung.

(5) mangelnde Ausgrenzbarkeit eines Risikos

(6) Schwierigkeiten interessentennaher Organisierung

(7) Notwendigkeit eines unmittelbar hoheitlichen Erbringens von sozialen Dienstleistungen

(I) Ein erstes Hindernis, das jede Institutionalisierung sozialer Sicherung überwinden muß, ist die mit der Herausbildung der bürgerlichen Gesellschaft entstandene sozialstrukturelle Individualisierung von an sich gesellschaftlichen Risiken ${ }^{6}$. Die Arbeitslosenversicherung durchbricht als eine Institution »vergesellschafteter « Problemlösung - wie alle anderen Versicherungen - diese Individualisierung von Risiken indem sie

- individuelle "Schuld « und Letztverantwortung in kollektive Verantwortung umdefiniert; sie drückt aus und bewirkt, daß Tatbestände gesellschaftlich und individuell nicht als »schicksalhaft " und allenfalls individuell vermeidbar erlebt werden, sondern sowohl in ihrer Verursachung wie in ihren Auswirkungen als gesellschaftlich beeinflußbar betrachtet werden (vgl. auch Böhle/Altmann 72, 6I);

- eine Strategie des "pooling the risks", eines Risikoausgleichs durch eine Konsolidierung von unterschiedlichen Risiken realisiert;

- eine nicht nur private Vergesellschaftungslösung wählt, wie z. B. den öffentlich gesetzten Versicherungszwang bei privaten Versicherungen (in der Art der Haftpflichtversicherung für Kraftfahrzeughalter), sondern eine öffentlich-rechtliche, der Form nach voll politisierte.

Dieses Durchbrechen der Individualisierung von sozialen Risiken erweitert und kanalisiert jene sozial-strukturellen Einbrüche, die zunächst durch eine Assoziierung von Arbeitern - die Bildung von Gewerkschaften - erzielt worden waren.

Die Bewegung hin zur Arbeitslosenversicherung kanalisierte so zwar die Forderungen der Gewerkschaften nach einer Beteiligung des Staates an ihren finanziellen Lasten bei der Versorgung von arbeitslosen Gewerkschaftsmitgliedern (vgl. Fuhrke/ Heimann 75, 147) allmählich in eine bürokratisch beherrschbare Form, allerdings unter Ubbernahme einer im Krisenfall weit schwieriger - weil nunmehr einer formellen politischen Verantwortlichkeit zurechenbar - zu begrenzenden Legitimationslast. Die Individualisierung von an sich gesellschaftlichen Risiken scheint im Fall der Arbeitslosigkeit insoweit eine besondere Kontur zu erhalten, als für dieses Risiko private Auffangelastizitäten stärker wirken konnten - so z. B. in der Familie und der agrarischen Feierabendproduktion (vgl. aber auch: Heinsohn/Knieper 74, 7y). Demgegenüber haben stärkere Beeinträchtigungen der Arbeitsfähigkeit (Krank-

6 Diese Individualisierung mußte sich gegenüber vorbürgerlichen kollektiven, personalisierten Verantwortungsformen (z. B. denen der Zünfte, des Herrn) durchsetzen und zerstörte diese Verantwortungsformen. Diese Individualisierung ist eine Folge der "Struktur der Privatheit “, die sich auch auf die sozialen Risiken erstreckt und der es entspricht, daß die bürgerliche Gesellschaft kein "Sozialmonopol des Staates « (Isensee 73, 74), des politischen Gemeinwesens kennt. 
heit, Unfall, Invalidität, Alter) schon früher private Auffangpotentiale überschritten.

(2) Eine weitere Barriere für die Institutionalisierung einer sozialen Versicherungsform ist gegeben, wenn der neu zu schaffende sozialrechtliche Regelungskomplex sich nicht als ein funktionales Äquivalent zu bestehenden Rechtsformen verfassen läßt. Die Arbeitslosenversicherung löst keine bestehenden Ansprüche ab. Sie ersetzt nur ansprucbsfreie Institutionen der gewerkschaftlichen Selbsthilfe, der kommunalen Armenfürsorge und der caritativen Organisationen. Etwas anderes gilt für die Unfallversicherung (so auch in den USA: vgl. Rimlinger $7 \mathrm{I}, 72 \mathrm{f}$; ; anders in England, a. a. O., S. 56 f.). Sie begrenzt und löst private Schadensersatzansprüche des Arbeiters gegen den Unternehmer ab, die dem Arbeiter aus Arbeitsvertrag (Verletzung der Fürsorgepflicht) und den den $\$ \$ 823 \mathrm{ff}$. BGB entsprechenden Bestimmungen ohnedies zustanden. Diese Barriere wirkt insoweit unabhängig von der Finanzwirksamkeit (s. (3)) als die neu zu schaffenden Rechtstitel, eine bürgerliche Struktur des »do ut des« und der Justiziabilität modifizieren müssen und damit quer zu den Konstruktionsprinzipien des bürgerlichen Rechtssystems und seiner Sanktionsinstanzen stehen.

Die Einführung der Arbeitslosenversicherung beinhaltet also, ebenso wie die der Kranken- und Rentenversicherung, eine strukturelle, sozialrechtliche Rechtsinnovation, weil sie kein funktionales Äquivalent zu bestehenden Rechtsformen darstellt und die Grenzen eines Rechtssystems überschreitet, das auf Individualisierung, Äquivalenz und Vertragsform aufgebaut ist.

(3) Der Umfang, in dem sozialpolitische Innovationen "finanzwirksam* sind, bedeutet ein weiteres Hindernis für die Institutionalisierung von Reformmaßnahmen. Die Arbeitslosenversicherung erfordert einen vergleichsweise hohen finanziellen Aufwand. Etwas anderes gilt z. B. für die Unfallversicherung, die zwar geringere aber ohnehin fließende Finanzströme in öffentliche Formen faßt und zudem innerhalb der Industrien die Bürden umverteilt. Es handelt sich bei der Einführung der Arbeitslosenversicherung also nicht um eine "Reform ohne Geld" (wie z. B. beim Erlaß von Arbeitsschutz- und Kündigungsschutzbestimmungen), sondern um eine sozialpolitische Innovation, die für die Lohnempfänger belastungsintensiv und von daher auch sozialstrukturell folgenreich ist.

(4) Auch ein $»$ Reichtum an sozialen Voraussetzungen (Komplexität) ist, wenn er das Fundament für sozialpolitische Innovationen darstellt, ein Hindernis für ihre Institutionalisierung.

Arm an sozialen Voraussetzungen - so gut wie voraussetzungslos - sind alle Reformen, die vornehmlich und nur das Bestehen einer bürgerlichen Gesellschaft voraussetzen, in welchem Entwicklungsstadium auch immer sie sich befinden mag, und ferner, einen gewissen öffentlich zu leistenden sozialstatistischen Überblick über das zu bearbeitende Problem. Kranken-, Renten- und Unfallversicherung sind in diesem Sinne voraussetzungslos. Sie benötigen als sozialen Anknüpfungspunkt - außer einem sozialstatistischen Uberblick - nur den Arbeitnehmerstatus, bestimmte versicherungsrelevante Eigenschaften (Krankheit usf.) und ein quasi steuerstaatliches, allgemeines Verfahren der Ressourcenabsorbtion, also Umstände, die ohne weiteres mit der Herausbildung der bürgerlichen Gesellschaft immer schon mitgesetzt sind.

Die Arbeitslosenversicherung hingegen ist - dies als erste zusätzliche soziale Voraussetzung - erst institutionalisierbar, wenn ein entwickelter nationaler Arbeitsmarkt existiert. Entwickelter nationaler Arbeitsmarkt heißt nicht, daß faktisch umfassende Mobilität und ein exakter Preisausgleich gegeben ist. Für einen entwikkelten nationalen Arbeitsmarkt reicht es aus, wenn der Preis der Ware Arbeitskraft 
in den Grundzügen überregional vereinheitlicht, umfassende Markttransparenz und umfassende Mobilität potentiell möglich ist. Erst dann ist ein System der Arbeitsvermittlung möglich und auch eine daran gekoppelte umfassende Unterstützung Arbeitsloser. Erst dann kann die Arbeitslosenversicherung als ein Regulator des Arbeitsmarkts funktionieren, sich auf ein vorgefundenes Preissystem (Tariflöhne) beziehen und es gestalten bzw. befestigen. Ohne die Entwicklung des nationalen Arbeitsmarkts konnte Arbeitslosigkeit nur punktuell im Wege der Armenhilfe aufgegriffen werden. Kurz: "Voraussetzung für die Arbeitslosenversicherung ist die vollkommen durchgeführte Organisierung des Arbeitsmarkts (Dr. Freund zitiert nach Wermel/Urban 49 I, 49; vgl. auch 25, 48). Ein solcher Arbeitsmarkt ist jedoch nicht schon mit der Herausbildung der bürgerlichen Gesellschaft gegeben. Zwar existiert schon bald ein solcher Markt (eine "Börse") für alle anderen Waren. Bildhaft gesprochen existierte für die Ware Arbeitskraft jedoch zunächst allenfalls ein Basar, eine Auktion (vgl. hierzu und zum folgenden: Lederer/Marschak 27, insbesondere $112-116)$. Es herrschte in diesem Bereich verglichen mit den anderen Warenmärkten eine Anarchie vor, die die Marktsynthese

- dem Arbeiter (über das Institut der »Umschau«),

- dem Zufall (Inseratwesen),

- einer auf enge Vermittlungsbereiche begrenzten, interessierten gewerblichen Stellenvermittlung oder

- dem Handeln eines Unternehmers mit je begrenztem, wenn auch relativ zu den Arbeitnehmern breiteren Marktüberblick

überließ (vgl. Lins 23, $824 \mathrm{ff}$.).

Erst in dem Maße wie die Individualisierung der Arbeiterschaft durch gewerkschaftliche Organisierung gebrochen wird, wie dann der Preis der Ware Arbeitskraft in verallgemeinerter Weise für ganze Branchen im nationalen Raum tarifiert werden kann und wie in der damit gesetzten Entwicklung eines nationalen Arbeitsmarktes die Hegemoniefrage über den »Arbeitsnachweis « zwischen Arbeitgeber- und Arbeitnehmerverbänden zum Problem wird, ergibt sich eine praktische Option für staatliches Handeln: die partielle Vergesellschaftung eines ja nunmehr vorhandenen, entwickelten nationalen Arbeitsmarktes durch das Einführen einer Arbeitslosenversicherung. Sie wurde dadurch ausgeübt, daß ein "Arbeitsvermittlungsmonopol « (vgl. heute die $\$ \$_{4}, 1_{3}, 18,228$ AFG) eingerichtet wurde.

Die Arbeitslosenversicherung ist ferner erst institutionalisierbar - dies als zweite zusätzliche soziale Voraussetzung - wenn die öffentliche Hand sich im Sinne eines positiven Garanten der kapitalistischen Entwicklung (z. B. im Sinne der Keynesianischen Wirtschaftspolitik) neu zu definieren beginnt. Zur Umdefinition von Unfall, Krankheit und Alter als soziale Risiken bedurfte es keiner derart weitreichenden wirtschaftspolitischen Systemmodifikation. Es genügte eine »humanitäre Umdefinition staatlichen Handelns, das sich allein auf die Bewältigung der negativen Folgen der kapitalistischen Entwicklung und nicht auf deren Hauptinstitutionen - Produktion, Arbeitsmarkt - beziehen mußte und das zudem durchaus in Übereinstimmung mit paternalistisch gefärbten vorbürgerlich-etatistischen Konzeptionen der Staatsfürsorge zu bringen war. Die Umdefinition von Arbeitslosigkeit als soziales Risiko bezieht sich jedoch auf eine Hauptinstitution der kapitalistischen Wirtschaft (den Zusammenhang der Klassen über den Arbeitsmarkt) und hat deshalb weitreichende Folgen für die gesamte staatliche Wirtschaftspolitik. Die Umdefinition bedeutet zudem einen Bruch mit der in Deutschland besonders lange wirksamen etatistischpaternalistischen Tradition des Fürsorgedenkens für die die personale Zuordnung der Arbeiter zum Arbeitgeber im Betrieb, nicht aber die anonyme Zuordnung über den Markt Ansatzpunkt von Reformen war. 
Im übrigen setzt die Arbeitslosenversicherung natürlich auch soziale Anknüpfungspunkte voraus, die mit der Entwicklung der bürgerlichen Gesellschaft ohne weiteres gesetzt sind (Arbeitnehmereigenschaft; Mittellosigkeit usf.). Sie ist auch schwerlich zu organisieren, ohne daß die entsprechenden Systemdaten systematisch erhoben würden?.

(5) Eine weitere Barriere für die Institutionalisierung sozialer Sicherung ist eine versicherungsmäßige Besonderheit des Risikos, die vor allem bei einer beschränkten Ausgrenzbarkeit des Risikos aus dem unmittelbaren staatlichen Verantwortungszusammenhang gegeben ist;

Die erste Besonderheit des Risikos der Arbeitslosenversicherung - verglichen mit den anderen sozialen Versicherungen - ist ihre versicherungsmäßige Strukturlosigkeit. Sie bedeutet für die öffentliche Hand eine besondere Belastung durch eine übermäßige Gefahr einer Politisierung dieses Tätigkeitsfeldes.

Unfälle, sowie Krankheits- und Rentenfälle sind jedenfalls grundsätzlich versicherungsstatistisch faßbar. Ihre Behandlung kann deshalb in abgegrenzten Risikogemeinschaften institutionalisiert werden. Die Errichtung solcher Gemeinschaften bringt über die strukturelle Innovation hinaus keine notwendigen weiteren Risiken einer Politisierung in großem Maßstab mit sich.

Bei dem Risiko "Arbeitslosigkeit ${ }^{8}$ ist die Lage jedoch anders. Dieses Risiko ist nicht versicherungsstatistisch faßbar und in abgegrenzter Risikogemeinschaft zu bannen, da es weder einen "zufälligen " noch einen "schätzbaren« Geldbedarf umfaßt $\mathrm{t}^{9}$. Eine Versicherung des »riskanten Status « Arbeitnehmer « birgt von daher immer schon das Risiko in sich, die legitimatorische Form der Versicherungsstruktur zu sprengen - eine Form, die ja gerade die Versicherbarkeit und damit die Individualisierbarkeit, die Begrenzbarkeit und die quantitative Beherrschbarkeit des "Schadens « suggerieren soll. Diese Sprengkraft zeigt sich beispielsweise, wenn die paritätischen "Beiträge « von Arbeitgeber und Arbeitnehmer zur Arbeitslosenversicherung, die insgesamt als Lohnbestandteile zu rechnen sind, politisch als quasi - Steuern verstanden und mit dem Hinweis darauf bekämpft werden, daß diese Ausgaben aus dem allgemeinen Staatshaushalt zu decken seien (vgl. z. B. Janzen, 75,4 f.; tendenziell ähnlich: Preller 70, 476). Von hierher erklärt sich auch die Forderung der Gewerkschaften, statt der Beiträge einen "Arbeitsmarktbeitrag " mit Steuercharakter zu erheben, der auch diejenigen Gruppen abgabepflichtig machen würde, die bislang zu den Kosten der Bundesanstalt für Arbeit nichts beigetragen haben, jedoch wie die Arbeitnehmer ein Interesse an einem funktionierenden Arbeitsmarkt besitzen, wie z. B. Beamte, Selbständige und Angehörige freier Berufe (vgl. Brück 75, i68).

Darüber hinaus ist zu beachten, daß das traditionell verstandene Risiko "Arbeitslosigkeit « nicht die gesamten Aufgabenbereiche der Bundesanstalt für Arbeit abdeckt. Als zusätzliche Maßnahmen gesellschaftspolitischen Zuschnitts ("Arbeitsmarktpolitik«) sind zu nennen: Maßnahmen der Beschäftigungspolitik, Berufliche Bildung, Rehabilitation, Verwirklichung der Chancengleichheit auf dem Arbeitsmarkt. Für diese Maßnahmen, die außerhalb des eigentlichen „Versicherungsrisikos « der Ar-

\footnotetext{
7 Die erste systematische Arbeitslosenzählung fand 1895 statt; vgl. Wermel/Urban I, 14.

$8 \mathrm{Vgl}$. insoweit auch BVerfGE 18,366, 376: "Die Arbeitslosenversicherung unterscheidet sich ihrem Wesen nach von anderen Zweigen der sozialen Versicherung dadurch, daß ihr Risiko in weitestem Umfang von der unvorhersehbaren Arbeitsmarktlage abhängt und daher einer Berechenbarkeit weniger zugänglich ist.“

$9 \mathrm{Vgl}$. auch Preller 70, 331 : „Der enge Zusammenhang aller Arbeitslosigkeit mit Wirtschaftspolitik und die Tatsache, daß in modernen Industriestaaten diese Wirtschaftspolitik weithin smanipuliert ‘ wird, läßt es zweifelhaft erscheinen, ob Arbeitslosigkeit als zzufällig bezeichnet werden kann und unzweifelhaft, daß sie nicht geschätzt, d. h. hier versicherungsmathematisch berechnet werden kann."
} 
beitslosigkeit liegen, gilt erst recht, daß sie nicht in eine abgegrenzte Risikogemeinschaft gebannt werden können.

Eine zweite Besonderheit des Risikos der Arbeitslosenversicherung ist die durchgängige unmittelbare Nähe des Versicherungsgegenstandes zum Arbeitsmarkt. Die verrechtlichte Arbeitslosenversicherung mit ihren klagbaren Leistungen setzt zu ihrer faktischen Wirksamkeit eine schon routinisierte Arbeitsmoral voraus (vgl. a. Wermel/Urban $49 \mathrm{I}, 5 \mathrm{r}$ ), die nur im Ausnahmefall in Frage gestellt ist und nachgeprüft bzw. "nachgebessert « werden muß. Das vergesellschaftete Risiko ist also besonders störempfindlich auf der Ebene individueller Leistungsmotivation und von daher schwer in eine "abgegrenzte " Institution zu bannen. Alle anderen Institutionen, auch die Krankenversicherung, haben keine derartig starke Arbeitsmarktnähe und sind deshalb von dieser Voraussetzung nicht abhängig und weniger störungsgefährdet.

(6) Ein weiteres Hindernis für die Institutionalisierung sozialer Sicherung ergibt sich dann, wenn die Notwendigkeit besteht, eine relativ "interessentenferne "Organisation zu schaffen, um eine mit der gegebenen Gesellschaftsstruktur noch vereinbare, wirksame Sicherung zu gewährleisten. Unfall- und Krankenversicherung können relativ interessentennah (als Berufsgenossenschaften; Betriebskrankenkassen, Ersatzkassen) organisiert werden, weil sie sozial nicht voraussetzungsreich sind (unkompliziert), weil die Risiken begrenzt sind und weil auf diese Weise Prävention und Schadensausgleich kombinierbar sind (Unfallversicherung).

Die öffentliche Arbeitslosenversicherung hingegen steht von Anfang an im sinteressentenferneren « Anziehungsbereich der zentralstaatlichen Agenturen öffentlicher Herrschaft und setzt eine institutionelle Tendenz, diese Agenturen stärker zu erweitern voraus (s. o. III (4)). Anders als bei den anderen Trägern sozialer Versicherung ist "Selbstverwaltung « hier stets ein Begriff gewesen, dem kaum ein soziales Substrat entspricht (vgl. Weller 69, 49 ff., 106 f., 155 f.; Bogs 73, 31-I I 2; Wertenbruch 75, 261 ff.). Die Selbstverwaltung im Bereich der Arbeitslosenversicherung dekorierte allenfalls eine faktische Subsumtion unter die jeweilige zentralstaatliche Arbeitsverwaltung. Die Arbeitslosenversicherung hat diese staatsnahe Tendenz, weil sie sozial voraussetzungsreich ist - sie ist ein konstitutives Element kontinuierlicher, zentralstaatlicher Arbeitsmarktpolitik - und weil das ihr zugeordnete Risiko strukturell grenzenlos ist - von daher kommt dem Einbau präventiver Sicherung ein besonderes Gewicht zu, z. B. in Form eines kontinuierlichen administrativen Durchgriffs des Zentralstaats in entscheidenden Fragen.

(7) Eine schließliche Barriere für die Institutionalisierung sozialer Sicherung ist es, wenn die Notwendigkeit besteht, unmittelbar hoheitlich nicht-bürokratische, immaterielle Dienstleistungen zu erbringen ${ }^{\circ}$. Das Erbringen von derartigen Dienstleistungen steht im Gegensatz zu einer Bürokratiestruktur des Typus legaler Herrschaft, wie sie auch heute noch den öffentlichen Raum dominiert. Um nichtbürokratische, immaterielle Dienstleistungen (wie Beratung, Vermittlung) als staatliche Aufgabe zu institutionalisieren bedarf es also eines besonderen Aufwands.

Die Kranken-, Unfall- und Rentenversicherungen ließen gegebenenfalls solche Dienstleistungen grundsätzlich durch Dritte - meist Private - erbringen (z. B. Arzte), sind also marktergänzende Institutionen. Soweit sie selbst Leistungen unmittelbar organisierten, handelte es sich nur

- um juristische, bürokratische Arbeit (Prüfung, ob ein Versicherungsfall vorliegt und ähnliches) oder

10 Zur Unvereinbarkeit von "politischer Produktion « und "bürokratischer Herrschaft " vgl. insbesondere Grauhan/Leibfried 77, 6y ff. Dort wird die hier nur skizzierte Begründung zu Punkt (7) weiter ausgeführt. 

Beiträgen.

Die Arbeitslosenversicherung läßt solche Dienstleistungen zwar auch durch Dritte erbringen (so wird die berufliche Bildung nur ausnahmsweise unmittelbar durch die Bundesanstalt organisiert) und ist selbst stark auf juristisch-bürokratische und monetäre Arbeitsvorgänge zentriert. Sie kann jedoch ohne Gefährdung ihres regulierenden Auftrags bestimmte immaterielle Dienstleistungen nicht abweisen oder ihnen nicht ausweichen:

- die Arbeitsvermittlung, die Arbeits- und Berufsberatung, die Lehrstellenvermittlung;

- das persönliche Eingehen auf die subjektiven Voraussetzungen für einen Zugang zur Gewährung von Geldleistungen, wie z. B.

- was ist verschuldete, was unverschuldete Arbeitslosigkeit,

- was ist zumutbare Arbeit, die gerade dieser Arbeitnehmer anzunehmen verpflichtet ist,

- die am Arbeitsmarkt orientierte Forschung.

Weil der Errichtung der Arbeitslosenversicherung - im Gegensatz zu allen anderen Versicherungsträgern - alle sieben Hindernisse entgegenstanden, war ein besonderer sachlicher und zeitlicher Institutionalisierungsaufwand erforderlich. Die verspätete Errichtung der Arbeitslosenversicherung - wie auch ihre Labilität - ist also kein zufälliges, sondern ein strukturellen Bedingungen zurechenbares Phänomen.

Literatur

Fritz Böble / Norbert Altmann, Industrielle Arbeit und soziale Sicherheit. Eine Studie über Risiken im Arbeitsprozeß und auf dem Arbeitsmarkt, Frankfurt (Athenäum) 1972

Harald Bogs, Die Sozialversicherung im Staat der Gegenwart. Offentlich-rechtliche Untersuchungen über die Stellung der Sozialversicherung im Verbändestaat und Versicherungswesen, Berlin (Duncker \& Humblot) I973

Gerhard Wilhelm Brück, Finanzierung der sozialen Sicherung, in: Gewerkschaftliche Monatshefte $3 / 75$, S. 159-168

Peter Flora u. a., On the Development of the Western European Welfare States, vv. Man., Mannheim August 1976

Jacques Fournier/ Nicole Questiaux, Traité du Social. Situations, luttes, politiques, institutions, Paris (Dalloz) 1976, S. 333-337, 472

Monika Fubrke / Siegfried Heimann, Das System der sozialen Sicherung in der BRD, in: Probleme des Klassenkampfes 19/20/21 (Oktober 1975), S. 145-170

Bentley B. Gilbert, British Social Policy 1914-1939, London (B. T. Batsford) 1970, S. 51-97 und $162-254$

ders., The Origins of the Welfare State. The Evolution of National Insurance in Great Britain. London (Michael Joseph) 1966, S. 233-288

John H. Goldthorpe, The Development of Social Policy in England, 1800-1914, Notes on a Sociological Approach to a Problem in Historical Explanation, in: Int. Soc. Association (ISA), Transactions of the 5 th World Congress of Sociology, Vol. VI, 1962, S. 4 I- 56

Rolf-Richard Grauhan / Stephan Leibfried, Die Sozialverwaltung zwischen politischer Herrschaft und politischer Produktion, in: Zeitschrift für Sozialreform 1977, S. 65-78

Gunnar Heinsohn / Rolf Knieper, Theorie des Familienrechts. Geschlechtsrollenaufhebung, Kindesvernachlässigung, Geburtenrückgang, Frankfurt (Suhrkamp) 1974

Josef Isensee, Umverteilung durch Sozialversicherungsbeiträge, Berlin (Duncker \& Humblot) 1973

Karl-Heinz Janzen, Sozialversicherung: Jeder sieht die Misere, in: Der Gewerkschafter, Heft $5 / 1975$, S. $4 \mathrm{ff}$.

Emil Lederer/Jacob Marschak, Die Klassen auf dem Arbeitsmarkt und ihre Organisationen, in: Grundriß der Sozialökonomik (Bearb. von G. Albrecht u. a.), IX. Abteilung, II. Teil, 
Die Autonomie und staatliche soziale Binnenpolitik im Kapitalismus, Tübingen (Mohr)

I927, S. $106-258$

Stephan Leibfried,Vorwort zu: Frances Fox Piven, Richard A. Cloward, Regulierung der Armut. Die Politik der öffentlichen Wohlfahrt, Frankfurt/Main (Suhrkamp) 1977, S. 9-67

W. Lins, Arbeitsmarkt und Arbeitsnachweis, in: Handwörterbuch der Staatswissenschaft, Jena 1923,4 . A., Bd. I, S. $824-839$

Roy Lubove, The Struggle for Social Security, 1900-1935, Cambridge, Mass. (Harvard University Press) i968, S. I44-177

John Mesher, Compensation for Unemployment, London (Sweet \& Maxwell) 1976

Daniel Nelson, Unemployment Insurance. The American Experience, 1915-1935. Madison etc. (University of Wisconsin Press) 1969

Ludwig Preller, Sozialpolitik in der Weimarer Republik, Stuttgart (Franz Mittelbach) 1949

Ludwig Preller, Praxis und Probleme der Sozialpolitik, Tübingen-Zürich (Mohr/Polygraph) 1970

Gaston V. Rimlinger, Welfare Policy and Industrialization in Europe, America, and Russia, New York usf. (John Wiley \& Sons) 197I

Günther Schmid, Steuerungssysteme des Arbeitsmarktes - Vergleich von Frankreich, Großbritannien, Schweden, DDR, Sowjetunion mit der Bundesrepublik Deutschland. Göttingen (O. Schwartz) 1975

Charles Evan Stewart, Unemployment Compensation - Response to a Crisis, in: Cornell Law Review (Bd. 61), S. $823-852$

Michael Stolleis (Hg.), Quellen zur Geschichte des Sozialrechts, Göttingen usf. (Musterschmidt) 1976

Florian Tennstedt, Sozialgeschichte der Sozialversicherung, in: Handbuch der Sozialmedizin (Maria Blohmke u. a., Hg.), Stuttgart (Enke) 1976, Bd. III, S. 385-492

Bernhard Weller, Arbeitslosigkeit und Arbeitsrecht. Untersuchung der Möglichkeiten zur Bekämpfung der Arbeitslosigkeit unter Einbeziehung der Geschichte des Arbeits- und Sozialrechts, Stuttgart (G. Fischer) 1969

Michael T. Wermel/ Roswitha Urban, Arbeitslosenfürsorge und Arbeitslosenversicherung in Deutschland, München (R. Pflaum Verlag) 1949, 3 Hefte

Wilhelm Wertenbruch, Gibt es noch Selbstverwaltung im Sozialrecht?, in: Die Sozialgerichtsbarkeit 1975 , S. $26 \mathrm{I}-269$ 